\title{
1 A multidimensional framework for studying social predation
}

\section{2 strategies}

3

4 Stephen D. J. Lang*a,b and Damien R. Farine*a,b,c

6 a Department of Collective Behaviour, Max Planck Institute for Ornithology, Konstanz, Germany

$7 \quad{ }^{b}$ Chair of Biodiversity and Collective Behaviour, Department of Biology, University of Konstanz, Konstanz,

8 Germany

9 c Edward Grey Institute of Field Ornithology, Department of Zoology, University of Oxford, U.K.

\section{ABSTRACT}

13 Social predation - the act of hunting and feeding with others - is one of the most successful life-

14 history traits in the animal kingdom. Though many predators hunt and feed together, a diversity of

15 mechanisms exist by which individuals forage socially. However, a comprehensive framework

16 capturing this diversity is lacking, preventing us from better understanding cooperative forms of

17 predation, and how such behaviours have evolved and been maintained over time. We outline a

18 framework of social predation that describes five key behavioural dimensions: sociality,

19 communication, specialisation, resource sharing, and dependence. By reviewing examples of social

20 predation, we demonstrate the strength of a multidimensional approach, highlighting key

21 commonalities and differences among species, and informative cross-dimensional correlations.

22 These patterns highlight different potential evolutionary pathways and end-points across a

23 multidimensional social predation spectrum.

25 Keywords: cooperation, foraging, hunting, group living, mutualism, predator-prey. 
30 Social predation allows groups of predators to find, target and kill larger or more numerous prey with greater efficiency than any individual can manage alone. Social foraging (searching for food) and social or group hunting (pursuing and capturing prey) provide many fitness benefits to individual predators; from enhanced prey detection [1] to the acquisition of additional resources [2$4]$, and is thought to be a key promoter of the evolution of group living $[5,6]$. For example, larger prides of lions (Panthera leo) are able to kill larger prey than smaller prides [7, 8]. Social predation is likely to have caused major shifts in the co-evolutionary arms race between predators and their prey [9]. Yet, while social predation has been repeatedly proposed as a classic case of cooperation, the mechanisms underpinning the process of social predation could be relatively simple, without the need for cooperation and instead arising via mutualisms and direct fitness benefits [10].

Despite extensive research on social predation, very little is known about how many times and in how many different forms it has evolved across taxa. Objective research has been restricted by the absence of a framework that quantifies the different forms of social predation. For example, groups containing individuals from more than one species can exhibit social predation behaviours that fit existing descriptions of cooperation [11-13] (see Box 1: Interspecific cases of social predation), thus highlighting limitations in the current approaches to describing and studying social predation. A robust framework is required to allow the similarities and differences among different groups of social predators to be more clearly evaluated. Such an evaluation would provide clearer insight into the evolutionary pathways that have led to the widespread strategies used by social predators throughout the animal kingdom.

We propose a novel framework to classify social predation among species according to five key dimensions of behaviour. Though the term 'social predation' has been previously used to "[cover the] complexities of finding prey and avoiding predation in groups" (and so including foraging behaviour of social herbivores) [14], here we limit it to include the range of different group hunting strategies used by social predators (animals that find, capture, and kill mobile prey animals). By extensively reviewing examples of social predation from the literature, and applying the framework to these examples, we demonstrate the strength of a multidimensional perspective. Our approach can identify commonalities in predation strategies across species, and linkages across dimensions. In creating this framework, we encourage experts on each species to report their findings using 
61 consistent and precise terminology. Such a framework will enable researchers to draw robust

62 comparisons of different species, facilitate greater focus for future research in a rapidly

63 modernising field (see Box 2 - How technology is advancing the understanding of animal

64 movement), and ultimately allow us to better understand the evolution of this diverse set of

65 behaviours.

66

2. EXISTING FRAMEWORKS OF SOCIAL PREDATION

Existing classification frameworks for social predation constitute one-dimensional continuums [15-

17]. Early studies focused their terminology on cooperation, defining it as a single behavioural trait [18-20] (hence the current common usage of 'cooperative hunting'). Boesch \& Boesch [15] were the first to outline a framework to delineate the "increasing complexity of organization between hunters". They based their ranked definitions of cooperation on how each individual of a group relates in time and space to its peers: showing similarity, synchrony, coordination, or collaboration (see Supplementary Table 1). Later, Ellis et al. [16] (Supplementary Table 2) and Bailey et al. [17] (Supplementary Table 3) outlined broader classification frameworks with additional hunting classes (Ellis' illustrated using raptors, Bailey's using Carnivora - though both are more widely applicable). These additions improved the Boesch \& Boesch framework by distinguishing the passive 'cooperation' of aggregated predators independently attracted to common resources, from the active cooperation among individuals in hunting groups. Both frameworks added greater consideration for different features of cooperation during hunting (resource sharing, division of 82 labour), and highlighted cases of pseudo-cooperative hunting.

\section{A MULTI-DIMENSIONAL PERSPECTIVE}

When formalizing social predation using one-dimensional frameworks that range from non-social foraging to cooperative hunting strategies $[15,16]$, it quickly becomes clear that current frameworks struggle to fully describe the diversity of social predation phenomena found in the animal kingdom (Box 3: Scoring with existing frameworks). Take the comparison of two social predator species currently classified as cooperative hunters: the lionfish (Dendrochirus zebra, taken from [21]), and the wolf (Canis lupus, taken from [22]). Socially, both species have distinct 
92 motivations for grouping: lionfish are commonly solitary, and form temporary groups with the main

93 purpose of foraging (though have been observed resting together [23]), whereas wolves live in

94 stable groups that live and hunt together. Their methods of hunt-initiating communication also

95 differ: lionfish flare their fins as an active signal to hunt together, whilst wolves appear to use the

96 behaviour of group conspecifics as passive cues to initiate hunting (but see [24]). During hunting,

97 lionfish alternate equally in specialist roles by taking turns at the front (where foraging success is

98 greatest), but wolves appear to hunt using collective movement rules $[22,25]$. Following a

99 successful hunt, wolves will divide the prey between pack members (according to dominance rank),

100 whereas individual lionfish aim to maximise their own intake. Clearly, each species' strategy

101 contains different elements; each with varying levels of complexity.

103 We propose using a framework that captures behaviour across multiple dimensions. Our aim is to 104 more accurately capture the range of behaviours associated with social predation, thus facilitating 105 clearer comparisons across taxa, and ultimately the identification of different potential

106 evolutionary pathways to social predation. This framework describes five fundamental dimensions 107 of social predation (see Figure 1), and is designed from the perspective of the individual and its 108 interactions with other group members (for a framework linking predator behaviour to prey size 109 and distribution, see [8]). Social predation behaviours for each dimension are divided into 110 subclasses, and ranked according to the increasing level of individual investment into actions that 111 maximize the benefits or success of the group. Below, we define the features of each dimension, 112 and illustrate them using taxonomically broad examples from the literature. Each dimension and 113 sub-section number relates to the framework outlined in Figure 1.

115 SOCIALITY (Interactions between individuals when foraging and feeding)

117 ASOCIAL (0) Solitary

118 Animals actively avoid or deter other individuals when foraging or feeding. Examples of this asocial 119 behaviour are common in animals that use discrete territories occupied by a single individual, like 120 tiger (Panthera tigris) [26], or pike (Esox lucius) [27], or by mated pairs that hunt separately, such as 121 sparrowhawk (Accipiter nisus) [28]. 
124 Predators forage solitarily but form aggregations at common resources. For example, annual

125 salmon runs draw large numbers of brown bears (Ursus arctos) to spawning grounds [29]. Although

126 animals in such aggregations appear to form temporary groups, each individual forages

127 independently.

\section{SOCIAL (2) Group foraging with inconsistent membership}

130 Individuals gain benefits from group-level collective foraging, which is thought to increase per-

131 capita success with group size [30]. Group membership however, is unstable. Such groups are

132 typically composed of unrelated individuals and have rapid membership turnover, exhibiting

133 fission-fusion dynamics [31]. Many examples also exist of interspecific groups forming for the

134 purpose of foraging (see Box 1), such as giant moray eels (Gymnothorax javanicus) and groupers

135 (Plectropomus pessuliferus) that have been observed hunting together [11]. While in some cases

136 the primary driver for forming groups is to reduce predation [6, 14] (typically mesopredators such

137 as piranhas or penguins avoiding superpredators [32-34]), grouping can also increase hunting

138 success [14].

\section{SOCIAL (3) Group foraging with consistent membership}

141 Individuals forage and feed in groups with consistent membership over time. Forming socially-

142 stable groups can reduce the level of agonistic interactions and increase predation success among

143 individuals. Groups can consist of unrelated individuals, and generally form in response to some

144 ecological pressure: for instance, improvements in the detection and capture of prey [14], or the

145 cooperative breeding and group caring of young [35]. Individual sperm whales (Physeter

146 macrocephalus) for example, have greater foraging success when hunting as groups, but studies

147 suggest their gregariousness is more likely to have evolved through mutual caring of calves [36-38].

148 Further, many animals that form socially stable groups also have sub-groups of individuals that hunt

149 for the group, which has been observed in several well-studied mammal groups. For instance, male

150 lions, and the females of some chimpanzee (Pan trogolodytes) groups rarely participate in hunts

151 [39-42] - though membership is still highly predictable in these hunting groups.

153 COMMUNICATION (Communication between individuals) 
Individuals do not modify their foraging behaviour in response to the behaviour of others.

NO SIGNALLING (1) Public/Social Information use

159 Group cohesion during foraging or at resources is driven by passive communication between

160 individuals. Social information (using cues generated by others, i.e. local enhancement) is a

161 mechanism that can lead to many emergent group-level properties [43,44], including maintaining

162 coordination [45]. Numerous animals choose between feeding sites based on the presence of

163 others, using visual [46-48] or auditory cues [49]. Even in several socially stable species, it appears

164 that individuals rely on simple passive behavioural cues to synchronise hunting $[22,39]$.

\section{SIGNAL (2) Recruitment/signalling at resources}

167 Predators use active signals to recruit or synchronise others at discovered food resources.

168 Recruitment of group members can enhance prey capture success and individual foraging efficiency

169 [50], or for mesopredators recruiting larger groups can help reduce individual vigilance for

170 superpredators [51]. The active signalling of food discovery can be via different modalities, but is

171 most frequently communicated using vocalisations [52]. For example, cliff swallows (Hirundo

172 pyrrhonota) that find insect swarms use vocal 'squeak calls' to signal conspecifics to feed [50].

173 Communication at discovered prey also appears to function as a means to synchronise prey capture

174 in certain groups of predators, such as packs of dhole (Cuon alpinus) [53] and schools of mormyrid

175 fish (Mormyrops anguilloides) [54].

SIGNAL (3) Recruitment/signalling to forage

178 Predators use active signalling to initiate foraging (prior to discovering resources). In African wild 179 dogs (Lycaon pictus), group members use a 'rally ceremony' to encourage other pack members to 180 start a hunt [55] whilst African penguins (Spheniscus demersus) employ a 'head-dipping' signal to 181 synchronise foraging dives [32]. In fish, groupers make a referential gesture to recruit moray eels $182[11,56]$, whilst lionfish flare their fins to recruit other individuals to their hunting parties [21].

183 Signals among predators are typically used for recruiting extra group members or to synchronise 184 initiation of the hunt. 
189 Individuals in groups (or aggregations) of predators forage and hunt independently. For example,

190 basks of Nile crocodiles (Crocodylus niloticus) near river crossings individually seize passing

191 ungulates [57]. Predators in such groups could have interaction rules that are associated with

192 avoiding collision with others [34,58]. However, the group's hunting success is not improved by

193 behavioural rules associated directly with foraging or hunting.

\section{NO SPECIALISATION (1) Individuals coordinate using identical collective rules}

196 Individuals in foraging or hunting groups use identical patterns of behaviour, forming a collective 197 group structure that benefits hunting success $[22,59,60]$. The coordination arising from the 198 collective interaction rules plays a key role in increasing the hunting success of the group [45]. Line-

199 formation is a common group-level strategy used by such collective predators, and can function to herd prey into dense groups and prevent escape $[60,61]$. Group hunting by predators has also been shown to facilitate the capture of collective prey by inhibiting collective information transfer within prey groups [62].

\section{SPECIALISATION (2) Temporary roles}

205 Animals coordinate with other group members, and some or all individuals take on different roles that function as part of a broader hunting strategy. Individually, these roles are adopted flexibly and can even be switched mid-hunt. For example, Harris' hawks (Parabuteo unicinctus) use a

208 hunting strategy with interchangeable 'chaser' and 'blocker' roles that function to flush and capture prey from dense vegetation [63]. Hunting strategies often have only one specialised position that is occupied by a random individual, while the rest of the group occupy a common role. In bottlenose dolphins (Tursiops truncatus) hunting schools of fish, group members take turns as lead 'chaser',

212 whilst conspecifics form a barrier to force fleeing fish to the surface [64]. Role specialisation is a

213 critical feature of group-level hunting strategies, distinguishing them from purely collective forms of 214 hunting.

\section{SPECIALISATION (3) Consistent roles}

217 Individuals consistently occupy the same specific role between hunts, and roles function as part of a

218 broader group-level strategy. For example, in lions, hunting females regularly occupy the left or

219 right side of a 'winging' hunting tactic [65], whilst individual chimpanzees consistently adopt a role 
of 'driver', 'blocker', 'chaser' or 'ambusher' over multiple hunts [66]. Role consistency should

221 enable individuals to practice and perfect role-specific motor control (the benefits of a consistent

222 attack technique has been shown in sailfish: individuals with stronger side-swipe lateralisation

223 experience higher capture success [67], although this example does not represent role

224 specialisation as part of a group-level hunting strategy). Studies on lions show that an individuals'

225 body type physiologically matches its role [65], suggesting that consistency also improves

226 development of role-specific muscle groups. Having consistent specialised roles in a family group

227 (as suggested in chimpanzees [66] and some killer whale ecotypes [68]) would also be more

228 effective in facilitating learning by offspring.

230 RESOURCE SHARING (Sharing of acquired resources among individuals)

COMPETITION (0) Individuals compete to maximize intake and monopolise resources

233 Predators compete to forage, and despotic individuals aim to monopolise resources by excluding all

234 others. Competitive resource division can be seen in numerous territorial species, such as

235 sparrowhawks [28].

\section{COMPETITION (1) Individuals divide resources among hunters according to effort}

238 Prey is divided amongst individuals via competition at the resource. Such behaviour is common in 239 predator species that forage on inherently divisible prey, such as sailfish (Istiophorus platypterus)

240 [69] and seabirds [70] feeding on fish schools, or bats [49] and swallows [50] feeding on insect 241 swarms.

\section{ALLOCATION (2) Individuals allocate according to social status (not competing)}

244 Once the prey is captured, it is allocated to group members according to social rank. For example, 245 when groups of mammalian carnivores including lions, African wild dogs or wolves capture large 246 prey, the resource becomes a feeding patch [17]. Larger, more aggressive or more dominant

247 individuals in the group can control access to the resource, and get preferential access [71].

248 Unequal resource sharing is common in animal groups governed by dominance hierarchies [72].

249 Similarly, if certain group members expended additional energy when hunting large prey, they are 250 allocated a larger share of the resulting resource. Examples of this type of resource division can be 251 seen in some chimpanzee populations [66] and human hunter-gatherer cultures [73]. 


\section{ALLOCATION (3) Individuals allocate according to need}

254 Individuals share the prey amongst all group members, allocating the resource in a way that maximizes benefit for all group members. In some cases, groups allocate resources to include individuals that did not participate in the hunt. It has also been suggested that sharing behaviour can occur in animal groups containing adults that are incapacitated as a result of illness or injury, such as pods of killer whales (Orcinus orca) [74] and neanderthal tribes (Homo neanderthalensis) 259 [75].

DEPENDENCE (Importance of social predation for hunting success of individual)

NO/MINIMAL DEPENDENCE (0) Pay a cost of foraging or hunting in groups (competition)

Having more than one predator has a detrimental effect on hunting success. For predators such as tiger [26], sparrowhawk [28], and pike [27] that use an ambush hunting strategy, the greatest success is achieved when hunting solitarily. Additional members increase the chance of groups being detected by prey, thus reducing hunting success.

\section{NO/MINIMAL DEPENDENCE (1) Neutral or small benefits - don't outweigh other costs}

Having more than one individual has a neutral or negligibly beneficial effect on success rate of the group. This is seen in bears aggregating at salmon runs [29], or piranhas shoaling around prey [34]. Any benefits that are accrued are a by-product of other factors (such as via reduced vigilance for mesopredators), and greater hunting ability is not the driving function of grouping.

Individuals gain greater benefits when hunting as a group. It is often the case that larger groups are more successful than smaller groups in detecting elusive prey [14], controlling collective prey (such as dolphins $[61,64]$ and whales $[76]$ that corral prey), and bringing down large prey (common in terrestrial carnivores [17]). 
283 Certain species are dependent on group hunting; in brown-necked ravens (Corvus rufficollis) [77],

284 individuals are only successful in catching lizards when hunting with others. In other cases,

285 individuals can hunt smaller species alone, but rely on hunting as a group to kill the prey required

286 to sustain reproduction [78]. In colonial insects like myrmicine ants (Pheidoiogeton diversus),

287 individuals cannot survive by hunting solitarily, as prey are too large for a single ant to subdue

288 alone [79].

\section{DESCRIBING SOCIAL PREDATION USING A MULTIDIMENSIONAL APPROACH}

294 The aim of the social predation framework is to provide novel insights compared to existing options. To test this, we first identified examples of social predation from the literature by searching Google Scholar with specific keywords (see Supplementary Methods for details). We identified 77 studies of social predation from four main vertebrate taxa that exhibit social predation (mammals, fish, birds and other reptiles). In addition, we selected six representative arthropod studies, and all six interspecific examples we could find (see Dataset 1 and Table 4 in

300 Supplementary Material for complete list). For each study, we scored group behaviour across the 301 five dimensions (Fig. 1) to identify their differences and commonalities in social predation features

302 (see Supplementary Table 1, and Supplementary Materials for methodology). In many species, 303 distinct populations could be scored differently across the five dimensions of the framework. Thus, 304 we demonstrate how the framework can also inform about within-species differences by including 305 multiple scores for killer whales. We calculated the variance in inter-observability by having two 306 independent scorers review 20 species, which resulted in only 5 out of 200 scores of differences 307 between the original (never differing by more than one dimension level).

309 To illustrate the strength of the proposed framework, we applied a dimension-reduction algorithm 310 (t-Distributed Stochastic Neighbour Embedding - or t-SNE [80]) to our scored data. t-SNE arranges

311 high-dimensional data points according to their similarity across dimensions (Figure 2). In our data,

312 the pairwise distance between points reflects the level of similarity in scores across all five

313 dimensions. We then applied a standard Gaussian mixture model clustering algorithm [81] to

314 identify clusters of similar species. While comparative studies would use the actual distances 
315 between each pair of species or populations, here we use clustering to provide a visual comparison

316 between the outputs of the new social predation framework and those of existing frameworks (see

317 Box 3).

319 Clustering of species (Figure 2) highlights the spread of interspecific examples across the diversity

320 of predation strategies, and how different populations of the same species can vary. First, we find

321 that most cases of interspecific social predation occur in the same cluster. These include

322 honeybadger (Mellivora capensis) with pale chanting goshawk (Melierax canorus), and wedge-

323 tailed shearwaters with skipjack tuna. These largely represent cases where one species benefits as

324 a by-product of the other species' hunting activity. By contrast, other interspecific examples are

325 spread across different clusters. The sharptooth catfish (Clarius gariepinus) hunting with blunttooth

326 catfish (Clarias ngamensis) is contained in a cluster containing yellowtail and bluefin tuna-both

327 species that can be described as coordinated hunters. Atlantic bottlenose dolphins hunting with

328 spotted dolphins sit in a cluster containing species such as red-bellied piranha and bank swallow

329 (Riparia riparia), which can be described as aggregative predators. More broadly, the distribution of 330 interspecific groups across these different clusters suggests that social predation strategies are not

331 all exclusively a result of complex kin-selected processes (see Box 1).

333 The clusters also highlight that different combinations of strategies can be used by different

334 populations of the same species. The social predation framework reveals that several ecotypes of

335 killer whales are found in separate clusters. Type B orca are clustered with species such as wild 336 dogs and humans, potentially representing the most advanced examples of social hunters. Two 337 other ecotypes (Transient and Resident) are found in the same cluster as Harris' hawks and dholes,

338 which are less-developed social hunters, that show high levels of resource sharing and minimal use 339 of active communication. In this context, the framework will be useful for quantifying how social 340 predation strategies vary across populations adapted for living in different ecological conditions 341 (such as different seasons, habitats, or prey type availability).

343 Clustering reveals both expected and unexpected results. For example, humans are found alongside 344 orcas, lions, and chimpanzees, but surprisingly, this cluster also contains Aplomado falcons and 345 myrmicine ants. Comparisons within and between clusters could be drawn with other 346 characteristics of each species, such as brain size or lifespan. However, the greatest strength of the 
347 framework will be to directly compare the scores within and among species across the different

348 dimensions, and we explore such comparisons in the following two sections. Further, future

349 research combining scores using the framework with phylogenetic information could identify

350 whether certain combinations of predation features represent convergent pathways to complex

351 social predation strategies, and examine the dependency structure linking these behaviours (see

352 Section 6: Dependencies across dimensions).

\section{CORRELATIONS BETWEEN DIMENSIONS}

Because of the inherent nature of social predation, as rank increases in one dimension it is also likely to increase in others (though not necessarily linearly or equally). We used a correlation analysis to explore in more detail the relationships between dimensions (see Supplementary Figure 1). We only found positive correlations, suggesting that all dimensions captured incremental complexity in similar ways. Further, none of the correlations were close to 1, which would have suggested that the framework needed to be collapsed to a smaller number of dimensions. Here, we

363 discuss the five sets of dimensions with correlations above 0.4 .

Sociality + Specialisation (0.52) - Role specialisation is likely to require repeated interactions between the same individuals. However, social predation in mixed-species social predator groups allows role specialisation as a species-level trait $[11,82]$ reducing the costs of finding individuals with the appropriate specialisation traits.

369 Sociality + Resource sharing (0.46) - Resource sharing requires individuals to reduce their own intake in lieu of others, such as when food is allocated to non-participants after hunts (e.g. [39,

371 66]). Such behaviour is most common when stable social groups are formed to increase

372 reproductive success.

373 Sociality + Dependence (0.56) - Living in stable social groups is likely to have enabled animal groups

374 to improve their hunting ability [5]. We hypothesise that increased pressure from predators could

375 induce responses from prey that include larger body sizes and larger groups. The coevolutionary

376 arms race that ensued could have trapped social predators into high dependence.

377 Specialisation + Resource sharing (0.4) - Specialisation when hunting large prey requires fair 378 allocation of the resource when a kill succeeds. Thus, resource allocation would be important in 
role-specialised strategies to ensure a mechanism to provide resources for individuals whose roles

380 do not place them near to captured resources (e.g. flushers).

381 Dependence + Specialisation (0.43) - Specialisation of roles when hunting in groups can improve the per capita capture of prey. Predators that use specialisation to improve hunting success could become dependent on these as their life histories become reliant on the higher resource balance specialised group hunting provides.

Overall, we find the strongest links between specialisation, resource sharing, and sociality. These links highlight potential traits that could have arisen as by-products of others. Such links raise key questions about the evolutionary origins of social predation (see Outstanding Questions), and the dependencies across the different dimensions.

\section{DEPENDENCIES ACROSS DIMENSIONS}

394 It is likely that dimensions of social predation have not evolved independently. Though we find that

395 dimensions largely represent different aspects of behavioural space, it is possible that some

396 behaviours first required others to evolve. Thus, only some evolutionary trajectories among the multitude of possible pathways could be possible (forming a tree-like structure). We explore the nature of such dependencies by searching for consistent differences among dimensions across all species. For each species we subtracted every pair of dimensional scores from one another, and plotted the results in histograms (see Supplementary Figure 2). The resulting graphs highlight that sociality appears to underpin all other traits (i.e. across all species, their sociality score is almost always higher than scores in other dimensions), whereas specialisation seems to be dependent on many of the other traits - notably sociality. It is therefore unlikely that specialisation would evolve

404 prior to sociality.

\section{IMPLICATIONS AND OUTLOOK}

409 Our framework provides a multi-dimensional approach that more completely describes all the 410 features of social predation. The framework provides guidelines for comprehensive and objective 
411 reporting of social predation by outlining keys areas of information that are needed to fully

412 describe the features of social predator systems. Further, our literature search revealed that many

413 different terms have been used in studies of group hunting. We propose 'social predation' as a

414 useful umbrella term to describe this diversity of behaviours, and offer a framework as a starting

415 point to operationalise it. As our ability to study social predators continues to improve, relating

416 findings back to the dimensions from this framework will enable further comparative research.

417 Future studies will be able to quantify social predation strategies of animals across taxa, or even

418 across populations of the same species, thus enhancing our understanding of the evolution of social 419 predation.

\section{ACKNOWLEDGEMENTS}

422 We thank Jacob Graving for suggesting the use of t-SNE to visualise the clustering of data points, 423 and for providing code for this. We also thank the Farine lab for input during conception of the 424 framework, Keith McMahon and Frederike Hilleman for acting as independent scorers, and Ralf 425 Kurvers, James Herbert-Read, Graeme Ruxton, Adam Wilkins and Ron Ydenberg for providing 426 valuable feedback on previous versions of this manuscript. We also thank Helder Hugo Dos Santos 427 for the illustration in Figure 2. This work was funded by the Max Planck Society.

\section{AUTHOR CONTRIBUTIONS}

430 SDJL conceived the original idea and collated all data used in analyses. SDJL and DRF outlined the 431 framework, conducted the statistical analyses, and wrote the manuscript.

\section{COMPETING FINANCIAL INTERESTS}

434 The authors declare no competing financial interests.

\section{MATERIALS AND CORRESPONDENCE}

437 All correspondence and material requests should be addressed to Stephen Lang

438 (slang@orn.mpg.de) and Damien Farine (dfarine@orn.mpg.de). 


\section{FIGURES}

443

444 Figure 1. Each dimension (grey box) is split into four subclasses, scored from 0-3 (coloured circles).

445 Each of the four sub-classes of a dimension can also be more broadly classified by presence or

446 absence of its key feature (social, signalling, specialisation, allocation, or dependence).

448 Figure 2. Dimensionality reduction of the scores across all five dimensions for 77 taxonomic

449 examples (see Supplementary Figure 3 for all species names). Each point reflects a specific animal

450 group scored using our social predation framework, and outlined letters refer to which taxonomic

451 group they belong to (see Box 1). The scores for each dimension are depicted by differently

452 coloured sections of the star plot, with the increasing size of each coloured section reflecting higher

453 scores (0-3). The dimensionality-reduction algorithm (t-SNE [80]) positions multi-dimensional data

454 points that are more similar closer to one another. The coloured background polygons are the

455 result of a Gaussian Mixture Model clustering algorithm [81] that is used to identify groups of

456 species with similar social predation strategies, enabling comparison to existing frameworks (see

457 Box 3).

458

459 


\section{BOX 1 - Interspecific cases of social predation:}

463 Consideration of social predation often comes with a tacit assumption that it requires complex

464 social mechanisms to evolve. However, there are many examples where individuals from different

465 species exhibit social predation behaviours. These suggest that the underlying processes facilitating

466 social predation can be relatively simple. Here we briefly review three examples of interspecific

467 social predation.

469 Commensalistic relationships can form between predator species when associations benefit 470 individuals from one species at no cost or benefit to heterospecifics. Ethiopian wolves (Canis 471 simensis), for example, associate with herds of gelada monkeys to form interspecific groups. Gelada 472 herds flush small rodents as they move through vegetation, and Ethiopian wolves have a higher 473 rate of prey capture when moving with baboon troops [83]. The wolves' strategy appears valuable 474 enough that they will forgo the chance to easily take juvenile geladas (resulting in expulsion) so that 475 they can retain the benefits of hunting within the group.

477 Interspecific groups can also mutualistically benefit all individuals involved. Several studies 478 investigating the associations between seabirds and subsurface predators have shown a higher rate 479 of prey capture for all individuals involved in heterospecific social predation, as each predator 480 species drives prey towards the other [70]. Examples include wedge-tailed shearwaters (Puffinus 481 pacificus) and skipjack tuna (Katsuwonus pelamis) that non-randomly associate with surface482 hunting fish [12]. Though generally facultative (shearwaters can also feed without heterospecifics), 483 there are cases of apparently obligate relationships. Frigate birds (Fregata spp.) and sooty tern 484 (Sterna fuscata) are rarely seen foraging independently of subsurface predators such as tuna [82].

486 Sophisticated interspecific relationships also exist where individuals use active communication and 487 have complementary hunting skills that maximize their hunting success when hunting as 488 heterospecific groups. One example is social predation by grouper and giant moray eel [11]. Using 489 body movements, groupers actively signal to morays to initiate foraging, and direct them to the 490 locations of prey. The specific hunting strategies of each species are complementary, with morays 491 flushing prey from crevasses and groupers flushing them into crevasses. The result is that hunting 
492 success for both groupers and moray eels is almost five times greater when hunting together than 493 when individuals hunt alone [11]. Such examples demonstrate that certain features of social 494 predation can be found in a range of heterospecific predator groups [84, 85], highlighting the 495 extent to which social predation behaviours can evolve from direct fitness benefits without 496 requiring indirect benefits such as kin selection [35]. 
499 Modern improvements to animal tracking technologies are enabling researchers to collect fine-

500 scale movement data over extended time periods [86]. Of particular relevance to the study of social 501 predation is the ability to simultaneously track multiple individuals at once. With high-resolution

502 positioning data collected from GPS tags, orientation data using magnometers, and movement data

503 collected using accelerometers, it is now possible to explore in detail some of the mechanisms that

504 underpin the group-level movement dynamics during group hunts.

506 Recent work investigating social decision-making in baboon troops used modern GPS-collars that collected high-accuracy $(0.5 \mathrm{~m})$ spatial data at high temporal resolution $(1 \mathrm{hz})$, allowing for comprehensive study of individual movements, and analysis of group-level collective outcomes [87]. Such tracking techniques can be effectively applied to social predator systems to study how group members initiate and coordinate hunts, and capture information about the amount of energy hunters expend under different conditions. For example, recent tracking studies on African wild dogs have used high-resolution GPS tags on all members of a pack to investigate the use of 514 be used to understand aspects of hunting behaviour not possible with conventional tags. For

515 instance, gaps in the understanding of how vocal communication influences hunting behaviour (see

516 Box 4: Outstanding questions) could be addressed using GPS tags capable of audio-recording 517 individual vocalisations. More generally, even though studies employing novel technology will rely 518 heavily on theory because of the challenges of carrying field experiments $[8,87,89]$, the ability to 519 conduct experiments will also be facilitated by technological advances (e.g. [90]).

521 One caveat of fitting GPS tags to predators is that the identity, behaviour and fate of prey is often 522 unknown. Future studies will be able to follow whole social groups of both predators and prey 523 simultaneously. This will allow researchers to better understand many aspects of the hunt - from 524 how predators select and pursue targets, the ultimate outcome of predation events, and the 525 downstream responses of prey to predation pressure. Previous research has already demonstrated 526 how combining tracking data from predators and prey can provide novel insights into how predator-prey dynamics shape ecosystem structure [89]. Non-invasive forms of animal tracking that 528 can locate and identify untagged individuals (for example drones) are also likely to become reliable, 529 cost-effective and practical methods to track groups of free-living animals [91-93]. In many cases, 
530 these technologies will not replace human observations, but will facilitate better quantification of

531 behaviour during hunts [94, 95].

532

533 Finally, combining long-term tracking of animals with modern methods for extracting data on the

534 hormonal profiles from passive samples (e.g. faecal) will yield greater insights into some of the

535 physiological mechanisms underlying group hunting behaviour, as well as prey responses. For

536 example, integrating such data with information from GPS tags about individual propensity to join

537 hunts, their role in the hunt (for example the effort expended or relative position), and the rate of

538 agonistic or affiliative behaviours towards others. Thus, recent technological developments in

539 animal tracking are making it possible to collect more information from a growing selection of

540 social predators and their prey-and for longer periods of time, thus gaining insights into both the

541 proximate and ultimate drivers of social predation.

542

543 Figure B2.1. Technology is facilitating new insights into social predation. GPS tags fitted to

544 predators, such as (a) African wild dogs (photo: J. Myatt [88]), and prey, such as (b) plains zebra

545 (photo: D. Rubenstein), will enable detailed studies into the mechanisms of the hunt. (c) Unmanned

546 aerial vehicles (UAVs) will enable simultaneous video tracking of predators and prey (photo: NOAA

547 fisheries, Vancouver Aquarium [93]), while other sensing technologies, such as (d) sonar (image:

548 N.O. Handegard [62]), can be used to track the interactions between predator and prey in more

549 visually-restricted tracking environments.

550 
552 All currently used classification schemes of social predation represent one-dimensional

553 continuums, ranging from passive or non-cooperative systems, to fully cooperative groups [15-17].

554 To evaluate the benefits of using a multi-dimensional approach to quantifying social predation

555 strategies, we identified clusters of species with similar combinations of behaviours in the

556 framework (coloured clusters in Figure 2). We then scored examples using two existing one-

557 dimensional schemes for defining cooperative hunting: Ellis [16] and Bailey et al. [17] (see

558 Supplementary Tables 1-2 for the detailed classification schemes). Combining these scores enabled

559 us to evaluate the differences between our multi-dimensional framework and current methods of

560 classifying social predation. If the social predation framework makes no improvements on the

561 existing classification methods, we would expect each of the clusters from Figure 2 to align with the

562 classification groups from the existing frameworks.

564 As shown by Figure B3.1, both the Ellis [16] and Bailey [17] frameworks appear to capture only the

565 extremes of the social predation spectrum (which corresponds with our findings from section 7).

566 These extremes are likely to represent what would normally be defined as cooperative social

567 predators (red and green), such as killer whales or chimpanzees; and largely non-social predators

568 (light blue and turquoise) like osprey or crocodiles. By contrast, other species that are clearly

569 clustered in the social predation framework are poorly represented in the existing frameworks, for

570 instance the light green, purple, and pink clusters. The extensive overlap of such clusters,

571 particularly following Bailey's [17] definitions, highlights how using a one-dimensional scheme only

572 partially describes the diversity of hunting strategies exhibited by some social predators.

574 Figure B3.1. Results of scoring each animal group according to the Bailey [17] (a) and Ellis [16] (b)

575 frameworks. Each row depicts a specific animal group. The column position of each point denotes

576 the category in which the example fits according to the focal classification scheme. Point colour

577 corresponds to the cluster assigned by the clustering algorithm used in Figure 2, and the intensity

578 of the background colour highlights the relative proportion of animal groups from the same cluster

579 in a single category.

580

581

582 
584 Examining the distribution of species scored using our framework has enabled us to identify both

585 gaps in the literature and key questions arising in the study of social predation.

587 Gaps:

588 Many studies of social predation only acknowledge the use of communication signals when these 589 are obvious, and the response to cues remains largely untested. As a result, the extent to which 590 groups of predators use active signals or pick up on the cues of others is not completely 591 understood. Wolves, for example, use vocalisations, body posture and olfactory signals to 592 communicate within and between groups [24,96], but the extent to which they use such signals in 593 the context of hunting - specifically to initiate hunts or synchronise attacks - remains poorly 594 understood. In mixed-species predator groups, it is unclear how much active communication occurs between species, and whether individuals actively target communication to heterospecifics.

597 Population-level differences appear to be an important source of variation in strategies among 598 groups of social predators. Recent studies support the idea that cultural evolution could play an 599 important population-level role in the formation and maintenance of social predation strategies 600 [97]. Further, very little is known about how such social predation strategies are improved or 601 adapted over time - both on an individual level and between generations [98]. The underlying 602 genetic and developmental mechanisms, as well as plasticity, associated with social predation 603 strategies remain unexplored.

605 Key questions arising:

606 The strong clustering emerging from our multi-dimensional framework raises the question of how 607 many different pathways exist for social predation to evolve. Several examples from very different 608 taxonomic groups demonstrate similar social predation strategies, whereas closely related species 609 can demonstrate widely divergent strategies (Figure 2). Thus, integrating data using the framework 610 into a phylogenetic analysis will yield novel insights into social evolution, including whether there is 611 any convergent evolution towards social predation strategies.

613 The strong correlation between sociality and multiple dimensions of the framework raises the 614 question of which traits evolved in response to selection and whether some traits arose as by- 
615 products. Which evolved first: the evolution of sociality that enabled predators to capture and 616 share large prey items, or the ability to capture large prey as groups that provided the foundations 617 for sociality? A notable area of research will be to study how social predation fits into predator-prey 618 coevolution, and to determine whether coevolution has trapped social predators into high 619 dependence.

620

621

622 


\section{GLOSSARY}

624

625 Aggregation - Gathering of individuals attracted to a common resource.

626 Collective - Groups with behaviours that arise from common inter-individual interaction rules.

627 - Coordinated (collective) - Individuals relate in time and space to actions of others.

628 - Synchronised (collective) - Individuals relate in time to actions of others.

629 Conspecifics - individuals of the same species.

630 Cooperative - Working with others (not always as equals).

631 Fission-fusion - Groups that change in individual membership over time.

632 Feeding - Consumption of a food resource.

633 Foraging - The act of searching for a food resource.

634 Group - Two or more individuals that respond to the behaviour of each other.

635 Hunting - Active searching, pursuit and capture of mobile prey animals.

636 Heterospecifics - Individuals of a different species.

637 Local enhancement - Attraction of an individual by the presence of others.

638 Mesopredator - A predator that is also preyed on by a superpredator.

639 Predator - An animal that derives (at least some of) its energy by consuming other mobile animals.

640 Social foraging - Searching for food (plants/animals) with others.

641 Social predation - Finding, capturing and consuming other animals with others.

642 Social predator - A predator that finds, capture and consume animals with others.

643 Socially-stable - Groups with social ties that persist in time and across contexts (feeding, breeding).

644 Superpredator - A predator than preys on mesopredators and/or other superpredators. 
649 1. Pitcher, T.J. et al. (1982) Fish in larger shoals find food faster. Behavioral Ecology and 650 Sociobiology $10(2), 149-151$.

651 2. Creel, S. (1997) Cooperative hunting and group size: assumptions and currencies. Anim Behav 54 652 (5), 1319-24.

653 3. Luhrs, M.L. et al. (2012) Strength in numbers: males in a carnivore grow bigger when they 654 associate and hunt cooperatively. Behavioral Ecology 24 (1), 21-28.

655 4. MacNulty, D.R. et al. (2014) Influence of group size on the success of wolves hunting bison. PloS 656 one $9(11)$, e112884.

657 5. Macdonald, D.W. (1983) The ecology of carnivore social behaviour. Nature 301 (5899), 379-384.

658 6. Krause, J. and Ruxton, G.D. (2002) Living in groups, Oxford University Press.

659 7. Schaller, G.B. (2009) The Serengeti lion: a study of predator-prey relations, University of Chicago 660 Press.

661 8. Packer, C. and Ruttan, L. (1988) The Evolution of Cooperative Hunting. The American Naturalist $662132(2), 159-198$.

663 9. Dawkins, R. and Krebs, J.R. (1979) Arms races between and within species. Proceedings of the 664 Royal Society of London B: Biological Sciences 205 (1161), 489-511.

665 10. Clutton-Brock, T. (2009) Cooperation between non-kin in animal societies. Nature 462 (7269), $66651-7$.

667 11. Bshary, R. et al. (2006) Interspecific communicative and coordinated hunting between groupers 668 and giant moray eels in the Red Sea. PLoS Biol 4 (12), e431.

669 12. Hebshi, A.J. et al. (2008) Associations between seabirds and subsurface predators around Oahu, 670 Hawaii. Aquatic Biology 19 (3), 89-98.

671 13. Elliser, C.R. and Herzing, D.L. (2016) Long-term interspecies association patterns of Atlantic 672 bottlenose dolphins,Tursiops truncatus, and Atlantic spotted dolphins, Stenella frontalis, in the 673 Bahamas. Marine Mammal Science 32 (1), 38-56.

674 14. Beauchamp, G. (2013) Social predation: how group living benefits predators and prey, Elsevier.

675 15. Boesch, C. and Boesch, H. (1989) Hunting behavior of wild chimpanzees in the Taï National Park. 676 American Journal of Physical Anthropology 78 (4), 547-573.

677 16. Ellis, D.H. et al. (1993) Social Foraging Classes in Raptorial Birds. BioScience 43 (1), 14-20.

678 17. Bailey, I. et al. (2012) Group hunting within the Carnivora: physiological, cognitive and

679 environmental influences on strategy and cooperation. Behavioral Ecology and Sociobiology 67 (1), 680 1-17.

681 18. Kruuk, H. (1972) The spotted hyena: a study of predation and social behavior, University of 682 Chicago Press.

683 19. Busse, C.D. (1978) Do chimpanzees hunt cooperatively? The American Naturalist 112 (986), 767684770.

685 20. Goodall, J. (1986) The chimpanzees of Gombe: Patterns of behavior, Belknap Press of Harvard 686 University Press.

687 21. Lonnstedt, O.M. et al. (2014) Lionfish predators use flared fin displays to initiate cooperative 688 hunting. Biol Lett $10(6)$.

689 22. Muro, C. et al. (2011) Wolf-pack (Canis lupus) hunting strategies emerge from simple rules in 690 computational simulations. Behav Processes 88 (3), 192-7.

691 23. Marsh, S.L., Fish fauna observations at the Kermadec Island Group (NZ), Tane, 1985.

692 24. Nowak, S. et al. (2006) Howling activity of free-ranging wolves (Canis lupus) in the Białowieża

693 Primeval Forest and the Western Beskidy Mountains (Poland). Journal of Ethology 25 (3), 231-237. 
694 25. Sumpter, D.J. (2006) The principles of collective animal behaviour. Philos Trans R Soc Lond B

695 Biol Sci 361 (1465), 5-22.

696 26. Sunquist, M.E. (1981) The social organization of tigers (Panthera tigris) in Royal Chitawan

697 National Park, Nepal, Smithsonian Institution Press Washington, DC, USA:.

698 27. Eklöv, P. (1992) Group foraging versus solitary foraging efficiency in piscivorous predators: the

699

700

701

702

703

704

705

706 perch, Perca fluviatilis, and pike, Esox lucius, patterns. Animal Behaviour 44, 313-326.

28. Newton, I. (2010) The sparrowhawk, A\&C Black.

29. Deacy, W. et al. (2016) Kodiak brown bears surf the salmon red wave: direct evidence from GPS collared individuals. Ecology 97 (5), 1091-1098.

30. Clark, C.W. and Mangel, M. (1986) The evolutionary advantages of group foraging. Theoretical population biology $30(1), 45-75$.

31. Silk, M.J. et al. (2014) The importance of fission-fusion social group dynamics in birds. Ibis 156 (4), 701-715.

707

708

709

710

32. Wilson, R.P. et al. (1986) Group Size in Foraging African Penguins (Spheniscus demersus).

Ethology 72 (4), 338-341.

33. Takahashi, A. et al. (2004) Synchronous diving behavior of Adélie penguins. Journal of Ethology 22 (1), 5-11.

711 34. Queiroz, H. and Magurran, A.E. (2005) Safety in numbers? Shoaling behaviour of the Amazonian

712

713

714

715

716

717 red-bellied piranha. Biology letters 1 (2), 155-157.

35. Dugatkin, L.A. (1997) The Evolution of Cooperation. BioScience 47 (6), 355-362.

36. Whitehead, H. (1989) Formations of foraging sperm whales, Physeter macrocephalus, off the

Galapagos Islands. Canadian journal of zoology 67 (9), 2131-2139.

37. Gordon, J.C.D., The behaviour and ecology of sperm whales off Sri Lanka, University of

Cambridge, 1987.

718

719

720

721

722

723

724

725

726

727

728

729

730

731

732

733

734

735

736

737

738

38. Whitehead, H. and Arnbom, T. (1987) Social organization of sperm whales off the Galapagos Islands, February-April 1985. Canadian Journal of Zoology 65 (4), 913-919.

39. Scheel, D. and Packer, C. (1991) Group hunting behaviour of lions: a search for cooperation. Animal Behaviour 41 (4), 697-709.

40. Boesch, C. (1994) Cooperative hunting in wild chimpanzees. Animal Behaviour 48 (3), 653-667.

41. Funston, P. et al. (2001) Factors affecting the hunting success of male and female lions in the Kruger National Park. Journal of Zoology 253 (4), 419-431.

42. Funston, P.J. et al. (1998) Hunting by male lions: ecological influences and socioecological implications. Animal Behaviour 56 (6), 1333-1345.

43. Berdahl, A. et al. (2013) Emergent sensing of complex environments by mobile animal groups. Science 339 (6119), 574-576.

44. Arganda, S. et al. (2012) A common rule for decision making in animal collectives across species. Proceedings of the National Academy of Sciences 109 (50), 20508-20513.

45. Herbert-Read, J. (2016) Understanding how animal groups achieve coordinated movement. Journal of Experimental Biology 219 (19), 2971-2983.

46. Krebs, J.R. (1974) Colonial nesting and social feeding as strategies for exploiting food resources in the Great Blue Heron (Ardea herodias). Behaviour 51 (1), 99-134.

47. Anderson, J.G. (1991) Foraging behavior of the American white pelican (Pelecanus

erythrorhyncos) in western Nevada. Colonial Waterbirds, 166-172.

48. Greene, E. (1987) Individuals in an osprey colony discriminate between high and low quality information. Nature 329 (6136), 239-241.

739 49. Dechmann, D.K. et al. (2010) Group hunting - a reason for sociality in molossid bats? PLoS One

$740 \quad 5(2)$, e9012. 
50. Brown, C.R. et al. (1991) Food-sharing signals among socially foraging cliff swallows. Animal

742 Behaviour 42 (4), 551-564.

743 51. Lima, S.L. and Dill, L.M. (1990) Behavioral decisions made under the risk of predation: a review

744

745 52. Clay, Z. et al. (2012) Food-associated vocalizations in mammals and birds: what do these calls

746 really mean? Animal Behaviour 83 (2), 323-330.

747

748

749

750

751

752

753

754

755

756

757

758

759

760

761

762

763

764

765

766

767

768

769

770

771

772

773

774

775

776

777

778

779

780

781

782

783

784

785

786

787

53. Fox, M.W. (1984) The whistling hunters: field studies of the Asiatic wild dog (Cuon alpinus), SUNY Press.

54. Arnegard, M.E. and Carlson, B.A. (2005) Electric organ discharge patterns during group hunting by a mormyrid fish. Proc Biol Sci 272 (1570), 1305-14.

55. Creel, S. and Creel, N.M. (1995) Communal Hunting and Pack Size in African Wild Dogs, LycaonPictus. Animal Behaviour 50 (5), 1325-1339.

56. Vail, A.L. et al. (2013) Referential gestures in fish collaborative hunting. Nat Commun 4, 1765.

57. Pooley, A.C. and Gans, C. (1976) The Nile crocodile. Scientific American 234 (4), 114.

58. Lukeman, R. et al. (2010) Inferring individual rules from collective behavior. Proceedings of the National Academy of Sciences 107 (28), 12576-12580.

59. Schmitt, R.J. and Strand, S.W. (1982) Cooperative Foraging by Yellowtail, Seriola lalandei

(Carangidae), on Two Species of Fish Prey. Copeia 1982 (3), 714-717.

60. Partridge, B.L. et al. (1983) The structure of schools of giant bluefin tuna in Cape Cod Bay.

Environmental Biology of Fishes 9 (3-4), 253-262.

61. Benoit-Bird, K.J. and Au, W.W. (2009) Cooperative prey herding by the pelagic dolphin, Stenella longirostris. J Acoust Soc Am 125 (1), 125-37.

62. Handegard, N.O. et al. (2012) The dynamics of coordinated group hunting and collective information transfer among schooling prey. Current biology 22 (13), 1213-1217.

63. Bednarz, J.C. (1988) Cooperative Hunting Harris' Hawks (Parabuteo unicinctus). Science 239 (4847), 1525-1527.

64. Gazda, S.K. et al. (2005) A division of labour with role specialization in group-hunting bottlenose dolphins (Tursiops truncatus) off Cedar Key, Florida. Proc Biol Sci 272 (1559), 135-40.

65. Stander, P.E. (1992) Cooperative Hunting in Lions - the Role of the Individual. Behavioral Ecology and Sociobiology 29 (6), 445-454.

66. Boesch, C. (2002) Cooperative hunting roles among Tai chimpanzees. Human Nature-an Interdisciplinary Biosocial Perspective 13 (1), 27-46.

67. Kurvers, R.H.J.M. et al. The Evolution of Lateralization in Group Hunting Sailfish. Current Biology.

68. Coscarella, M.A. et al. (2015) Short Note: Technique Used by Killer Whales (Orcinus orca) When Hunting for Dolphins in Patagonia, Argentina. Aquatic Mammals 41 (2), 192-197.

69. Herbert-Read, J.E. et al. (2016) Proto-cooperation: group hunting sailfish improve hunting success by alternating attacks on grouping prey. Proceedings of the Royal Society B: Biological Sciences 283 (1842).

70. Thiebault, A. et al. (2016) How to capture fish in a school? Effect of successive predator attacks on seabird feeding success. J Anim Ecol 85 (1), 157-67.

71. Holekamp, K.E. and Smale, L. (1990) Provisioning and food sharing by lactating spotted hyenas, Crocuta crocuta (Mammalia: Hyaenidae). Ethology 86 (3), 191-202.

72. Janson, C. (1985) Aggressive competition and individual food consumption in wild brown capuchin monkeys (Cebus apella). Behavioral Ecology and Sociobiology 18 (2), 125-138.

73. Alvard, M.S. (2003) Kinship, lineage, and an evolutionary perspective on cooperative hunting groups in Indonesia. Human Nature 14 (2), 129-163. 
74. Wright, B.M. et al. (2016) Kin-directed food sharing promotes lifetime natal philopatry of both sexes in a population of fish-eating killer whales, Orcinus orca. Animal Behaviour 115, 81-95. 75. Spikins, P. et al. (2014) The Cradle of Thought: Growth, Learning, Play and Attachment in Neanderthal Children. Oxford Journal of Archaeology 33 (2), 111-134.

76. Wiley, D. et al. (2011) Underwater components of humpback whale bubble-net feeding behaviour. Behaviour 148 (5), 575-602.

77. Yosef, R. and Yosef, N. (2009) Cooperative hunting in Brown-Necked Raven (Corvus rufficollis) on Egyptian Mastigure (Uromastyx aegyptius). Journal of Ethology 28 (2), 385-388.

78. Hill, K. and Hurtado, A.M. (2009) Cooperative breeding in South American hunter-gatherers. Proc Biol Sci 276 (1674), 3863-70.

79. Moffett, M.W. (1988) Foraging dynamics in the group-hunting myrmicine ant, Pheidologeton diversus. Journal of Insect Behavior 1 (3), 309-331.

80. Maaten, L.v.d. and Hinton, G. (2008) Visualizing data using t-SNE. Journal of Machine Learning Research 9 (Nov), 2579-2605.

81. Fraley, C. et al. (2012) Normal mixture modeling for model-based clustering, classification, and density estimation. Department of Statistics, University of Washington 23, 2012.

82. Au, D.W. and Pitman, R.L. (1988) Seabird relationships with tropical tunas and dolphins. Seabirds and other marine vertebrates. Columbia University Press, New York, 174-212.

83. Venkataraman, V.V. et al. (2015) Solitary Ethiopian wolves increase predation success on rodents when among grazing gelada monkey herds. Journal of Mammalogy 96 (1), 129-137.

84. Spottiswoode, C.N. et al. (2016) Reciprocal signaling in honeyguide-human mutualism. Science 353 (6297), 387-389.

85. Minta, S.C. et al. (1992) Hunting associations between badgers (Taxidea taxus) and coyotes (Canis latrans). Journal of Mammalogy 73 (4), 814-820.

86. Kays, R. et al. (2015) Terrestrial animal tracking as an eye on life and planet. Science 348 (6240), aaa2478.

87. Strandburg-Peshkin, A. et al. (2015) Shared decision-making drives collective movement in wild baboons. Science 348 (6241), 1358-1361.

88. Hubel, T.Y. et al. (2016) Additive opportunistic capture explains group hunting benefits in African wild dogs. Nature communications 7.

89. Ford, A.T. et al. (2014) Large carnivores make savanna tree communities less thorny. Science 346 (6207), 346-9.

90. Firth, J.A. et al. (2016) Pathways of information transmission among wild songbirds follow experimentally imposed changes in social foraging structure. Biology letters 12 (6), 20160144. 91. Christie, K.S. et al. (2016) Unmanned aircraft systems in wildlife research: current and future applications of a transformative technology. Frontiers in Ecology and the Environment 14 (5), 241251.

92. Harvey, R.J. et al. (2016) Determining position, velocity and acceleration of free-ranging animals with a low-cost unmanned aerial system. Journal of Experimental Biology.

93. Durban, J. et al. (2015) Photogrammetry of killer whales using a small hexacopter launched at sea 1. Journal of Unmanned Vehicle Systems 3 (3), 131-135.

94. Williams, T.M. et al. (2014) Instantaneous energetics of puma kills reveal advantage of felid sneak attacks. Science 346 (6205), 81-85.

95. Wilson, R.P. et al. (2015) Mass enhances speed but diminishes turn capacity in terrestrial pursuit predators. Elife 4, e06487.

96. Mech, L.D. and Boitani, L. (2010) Wolves: behavior, ecology, and conservation, University of Chicago Press. 
835 97. Foote, A.D. et al. (2016) Genome-culture coevolution promotes rapid divergence of killer whale 836 ecotypes. Nat Commun 7, 11693.

837 98. Biro, D. et al. (2016) Bringing a Time-Depth Perspective to Collective Animal Behaviour. Trends 838 Ecol Evol 31 (7), 550-62.

839 